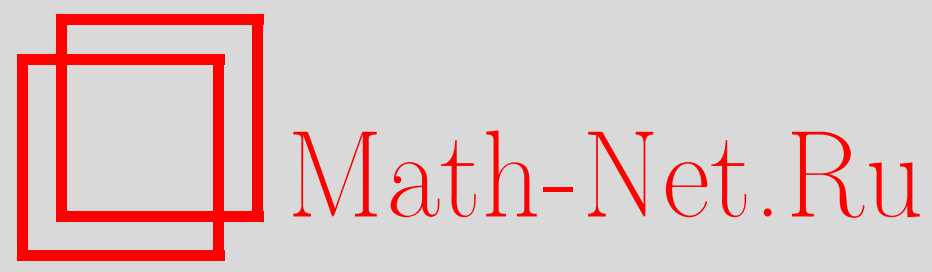

А. А. Феликсон, Кубы Ламберта, порождающие дискретные группы отражений, Матем. заметки, 2004, том 75, выпуск 2, 277-286

DOI: https://doi.org/10.4213/mzm14

Использование Общероссийского математического портала Math-Net.Ru подразумевает, что вы прочитали и согласны с пользовательским соглашением http://www.mathnet.ru/rus/agreement

Параметры загрузки:

IP : 54.80 .97 .219

26 апреля 2023 г., $14: 37: 12$ 


\section{КУБЫ ЛАМБЕРТА, ПОРОЖДАЮЩИЕ ДИСКРЕТНЫЕ ГРУППЫ ОТРАЖЕНИЙ}

\section{А. А. Феликсон}

Кубом Ламберта $Q(\alpha, \beta, \gamma)$ называется куб, все двугранные углы которого прямые, за исключением углов $\alpha, \beta$ и $\gamma$ при трех ребрах, никакие два из которых не лежат в одной плоскости. В данной работе мы классифицируем кубы Ламберта в $S^{3}, \mathbb{E}^{3}$ и $\mathbb{H}^{3}$ такие, что группа $G_{Q}$, порожденная отражениями относительно граней куба $Q$, дискретна.

Библиография: 8 названий.

Введение. Пусть $P$ - вьпуклый многогранник в трехмерном сферическом пространстве $S^{3}$, евклидовом пространстве $\mathbb{E}^{3}$ или гиперболическом пространстве $\mathbb{H}^{3}$. Рассмотрим группу $G_{P}$, порожденную отражениями в гранях многогранника $P$. Будем называть $G_{P}$ группой отражений, порожденной многогранником $P$. Задача состоит в перечислении многогранников, порождающих дискретные групы отражений.

Несмотря на более чем вековую историю вопроса, ответ известен только для многогранников простейших комбинаторных типов. В 1873 г. Шварц [1] перечислил треугольники на сфере, порождающие дискретные группы отражений. В 1998 г. Клименко и Сакума [2] решили задачу для гиперболических треугольников. В [3]-[5] задача решена для гиперболических четырехугольников, ограниченных гиперболических пирамид и треугольных призм, а также для гиперболических симплексов.

В данной работе задача решена для кубов Ламберта в $S^{3}, \mathbb{E}^{3}$ и $\mathbb{H}^{3}$.

ОПРЕДЕЛЕНИЕ 1. Кубом Ламберта $Q(\alpha, \beta, \gamma)$ называется многогранник, комбинаторно эквивалентньй кубу, все двугранные углы которого прямые, за исключением углов $\alpha, \beta$ и $\gamma$ при трех ребрах, никакие два из которых не лежат в одной плоскости.

ОПРЕДЕЛЕНИЕ 2. Многогранник $P$ назьвается многогранником Кокстера, если все его двугранные углы являются целыми частями $\pi$.

В п. 1 доказано, что сферический куб Ламберта $P$ порождает дискретную грушу отражений тогда и только тогда, когда $P=Q(2 \pi / 3,2 \pi / 3,3 \pi / 4)$. В пп. 2 и 3 доказано, что куб Ламберта в евклидовом или гиперболическом пространстве порождает дискретную группу отражений тогда и только тогда, когда $P$ является многогранником Кокстера.

Работа выполнена при поддержке Национального научного фонда Швейцарии, грант № 20-61379.00. 
Основные свойства и обозначения. Предположим, что $P$ является многогранником Кокстера. Тогда группа $G_{P}$ дискретна и $P$ является ее фундаментальной областью.

Предположим, что многогранник $P$ не кокстеровский и групша $G_{P}$ дискретна. Тогда существует конечное число таких элементов $\gamma \in G_{P}$, что многогранник $\gamma P$ пересекается с многогранником $P$. При этом зеркала групшы $G_{P}$ разбивают $P$ на конечное число частей (плоскость назьвается зеркалом группы $G_{P}$, если $G_{P}$ содержит отражение относительно данной плоскости).

ОПРЕДЕЛЕНИЕ 3. Кокстеровским разбиением вьпуклого многогранника назьвается такое его разбиение на конечное число многогранников Кокстера, что любые два многогранника разбиения, имеющие общую грань, симметричны друг другу относительно нее.

Многогранники разбиения назьваются фундаментальными многогранниками кокстеровского разбиения.

Легко видеть, что любой некокстеровский многогранник $P$, порождающий дискретную групшу отражений, допускает нетривиальное кокстеровское разбиение. Пусть $F-$ фундаментальный многогранник этого разбиения. Тогда группу $G_{P}$ можно описать как группу $G_{F}$, порожденную многогранником Кокстера $F$.

Далее в настоящей работе рассматриваются лиш многогранники $P$, для которых группа отражений $G_{P}$ дискретна.

Многогранники Кокстера будем представлять с помошью их схемы Kокстера: вершины $v_{1}, \ldots, v_{k}$ схемы соответствуют граням $f_{1}, \ldots, f_{k}$ многогранника, вершины $v_{i}$ и $v_{j}$ соединены ребром кратности $k-2$, если двугранньй угол между гранями $f_{i}$ и $f_{j}$ равен $\pi / k$ (если грани $f_{i}$ и $f_{j}$ взаимно ортогональны, то вершины $v_{i}$ и $v_{j}$ не соединены).

Для сферических тетраэдров Кокстера мы используем стандартные обозначения (см. рис. 1)

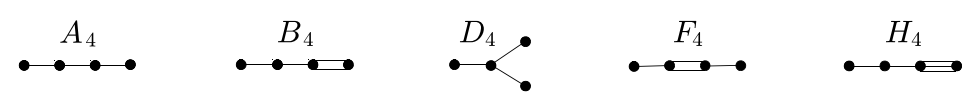

Рис. 1. Сферические тетраэдры Кокстера

1. Сферические кубы Ламберта. В данном пункте доказана

Теорема 1. Сферический куб Ламберта $P$ порождает дискретную группу отражсений тогда и только тогда, когда $P=Q(2 \pi / 3,2 \pi / 3,3 \pi / 4)$.

Сферический многогранник Кокстера, не содержащий двух противолежащих точек сферы $S^{3}$, является тетраэдром. Поэтому группа отражений $G_{P}$, порожденная кубом $P$, является в то же время группой отражений $G_{T}$, порожденной некоторьп сферическим тетраэдром Кокстера $T$. Ясно, что каждая грань куба $P$ лежит на некотором зеркале группы $G_{T}$.

Будем говорить, что многогранник $T$ разбивает многогранник $P$ (или $P$ paзбивается многогранником $T$ ), если группа отражений $G_{P}$ является подгруппой группы отражений $G_{T}$.

Лемма 1. Пусть T- сферический тетраэдр Кокстера. Предположим, Т разбивает куб. Тогда схема Кокстера $\bar{T}$ тетраэдра $Т$ связна. 
ДокАЗАтЕльство. Предположим, что $\bar{T}$ не связна. Тогда либо в $\bar{T}$ есть вершина, не соединенная ни с какой другой вершиной, либо $\bar{T}=G_{2}^{(m)}+G_{2}^{(n)}$.

Предположим, что в $\bar{T}$ есть вершина $v$, не соединенная ни с какой другой вершиной. Пусть $v$ соответствует грани $f$ тетраэдра $T$ и $A$ - вершина тетраэдра $T$, противолежащая грани $f$. Тогда грань $f$ перпендикулярна остальным граням тетраэдра. Поэтому любое зеркало групшы $G_{T}$, не содержащее грани $f$, проходит через точку $A$. Отсюда следует, что любой простой многогранник, которьй разбивается симплексом $T$, имеет не более четырех граней. Это невозможно, поскольку $T$ разбивает куб.

Предположим, что $\bar{T}=G_{2}^{(m)}+G_{2}^{(n)}$. Пусть $\bar{l}_{1}$ - пересечение пары не ортогональных друг другу граней тетраэдра $T$ и $l_{1}-$ прямая, содержащая ребро $\bar{l}_{1}$. Пусть $\bar{l}_{2}$ и $l_{2}-$ ребро и прямая, соответствуюшие второй паре взаимно не ортогональных граней. Легко видеть, что любое зеркало групшы $G_{T}$ содержит либо $l_{1}$, либо $l_{2}$. Поэтому любой многогранник, которьй разбивается тетраэдром $T$, имеет не более четырех граней и, следовательно, не может быть кубом.

Отсюда следует, что если $T$ разбивает куб, то $T$ является одним из тетраэдров $A_{4}$, $B_{4}, D_{4}, F_{4}$ или $H_{4}$.

ЛЕмма 2. Пусть $t$ - треугольник с углами $(\pi / 2, \pi / 2, \pi / 3)$ или $(\pi / 2, \pi / 3, \pi / 3)$. Тогда $t$ не разбивает правильного прямоугольного треугольника $p$.

Пусть $t$ - треугольник с углами $(\pi / 2, \pi / 2, \pi / 2)$ или $(\pi / 2, \pi / 3, \pi / 3)$. Тогда $t$ не разбивает треугольника р с углами $(\pi / 2, \pi / 2,2 \pi / 3)$.

ДокАЗАТЕЛьСтво. Для доказательства леммы достаточно показать, что $G_{p}$ не является подгруппой в $G_{t}$. Подгрупш, порожденные отражениями в сферических группах, порожденных отражениями, перечислены Кокстером (см. [6]).

Лемма 3. Пусть $T=A_{4}$ или $T=D_{4}$. Тогда $T$ не разбивает куба Ламберта.

ДокАЗАтЕЛЬСтво. Предположим, что $T$ разбивает куб Ламберта $P$. Тогда $P$ допускает кокстеровскоеразбиение с фундаментальным тетраэдром $T$. Пусть $A$-вершина куба $P$, в которой сходятся три взаимно перпендикулярных грани. Пусть $s-$ малая сфера с центром в $A$. Тогда пересечение $s$ и $P$ является правильным прямоугольным треугольником $p$. Пусть $T_{1}$ - фундаментальньй тетраэдр, лежащий в $P$ и имеющий вершину $A$. Тогда пересечение $s$ и $T_{1}$ является треугольником $t_{1}$ и $t_{1}$ разбивает правильньй прямоугольньй треугольник $p$.

Пусть $T=A_{4}$. Из первой части леммы 2 следует, что $t_{1}$ не разбивает правильных прямоугольных треугольников. Противоречие доказьвает лемму для случая $T=A_{4}$.

Пусть $T=D_{4}$. Тогда один из двугранных углов куба $P$ равен $2 \pi / 3$ (иначе $P$ является сферическим кубом Кокстера, что невозможно). Поэтому куб Ламберта $P$ имеет такую вершину $A$, что пересечение $P$ с маленькой сферой с центром в $A$ является треугольником $(\pi / 2, \pi / 2,2 \pi / 3)$. Это противоречит второй части леммы 2.

Таким образом, любой куб Ламберта, порождающий дискретную группу отражений, разбивается одним из тетраэдров $B_{4}, F_{4}$ или $H_{4}$. Поскольку $G_{B_{4}}$ является подгруппой группы $G_{F_{4}}$, достаточно перечислить кубы Ламберта, которые разбиваются тетраэдрами $F_{4}$ и $H_{4}$.

Лемма 4. Тетраэдр $F_{4}$ разбивает ровно один куб Ламберта, а именно куб $Q(2 \pi / 3,2 \pi / 3,3 \pi / 4)$. 
ДоказАтельство. Рассмотрим $S^{3}$ как единичную сферу в $\mathbb{E}^{4}$. Пусть $e_{1}, e_{2}, e_{3}, e_{4}-$ ортонормированньй базис в $\mathbb{E}^{4}$. Пусть $\Delta$ - система корней с группой Вейля $G_{F_{4}}$. Тогда

$$
\Delta=\left\{ \pm e_{i}, \pm e_{i} \pm e_{j}, \frac{1}{2}\left( \pm e_{1} \pm e_{2} \pm e_{3} \pm e_{4}\right)\right\}
$$

Любое зеркало группы $G_{F_{4}}$ соответствует вектору в $\Delta$, ортогональному к этому зеркалу.

Куб Ламберта $P$ можно представить как пересечение единичной сферы и шестигранного конуса, все грани которого проходят через начало координат. Поэтому $P$ определяется внешними нормалями $f_{1}, f_{2}, \ldots, f_{6}$ к граням конуса, т.е. набором из шести векторов, лежащих в $\Delta$. Обозначим через $f_{i}=0$ грань конуса с вектором нормали $f_{i}$, $i=1,2, \ldots, 6$.

Для того чтобы шесть векторов определяли комбинаторный куб, эти векторы должны удовлетворять следующим условиям:

(1) вектор $f_{i}$ не пропорционален $f_{j}$ при $i \neq j$;

(2) система неравенств $\left(f_{i}, x\right)<0, i=1, \ldots, 6$, имеет решение - иначе нет многогранника, соответствующего векторам $f_{i}$ (здесь $x$ - вектор, а $(\cdot, \cdot)$ - скалярное произведение);

(3) пусть $f_{1}=0$ - грань конуса, противолежашая грани $f_{4}=0$; тогда система

$$
\left\{\begin{array}{l}
\left(f_{1}, x\right)=0, \\
\left(f_{4}, x\right)=0, \\
\left(f_{i}, x\right)<0, \quad i \neq 1, \quad i \neq 4
\end{array}\right.
$$

не имеет решений (иначе грани $f_{1}=0$ и $f_{4}=0$ пересекаются на единичной сфере и не соответствуют противолежащим граням куба).

Для того чтобы полученный куб был кубом Ламберта, векторы должны удовлетворять дополнительному условию:

(4) $\left(f_{i}, f_{j}\right)=0$, если пара $(i, j)$ отлична от $(1,6),(2,4),(3,5)$ (мы считаем, что грань $f_{1}=0$ противоположна грани $f_{4}=0$, грань $f_{2}=0$ противоположна грани $f_{5}=0$, a грань $f_{3}=0-$ грани $\left.f_{6}=0\right)$.

Для доказательства леммы достаточно проверить эти условия для каждой шестерки векторов из $\Delta$. Проверка проведена с помошью перебора. При этом найдена ровно одна (с точностью до симметрии) шестерка векторов, удовлетворяющая всем условиям:

$$
\begin{array}{ll}
f_{1}=e_{1}, & f_{4}=\frac{1}{2}\left(-e_{1}+e_{2}-e_{3}-e_{4}\right), \\
f_{2}=e_{2}, & f_{5}=-e_{2}-e_{4}, \\
f_{3}=e_{3}-e_{4}, & f_{6}=e_{1}-e_{3} .
\end{array}
$$

Куб Ламберта, определяемый этими векторами, равен $Q(2 \pi / 3,2 \pi / 3,3 \pi / 4)$. Группа, порожденная этим кубом, совпадает с $G_{F_{4}}$. 
ЗАмЕчАниЕ. Для уменьшения перебора можно зафиксировать три взаимно ортогональных вектора $f_{1}, f_{2}$ и $f_{3}$. С точностью до симметрии существует только четыре таких тройки векторов:

$\begin{array}{llll}\text { 1) } e_{1}, & e_{2}, & e_{3} ; \\ \text { 2) } e_{1}, & e_{2}, & e_{3}-e_{4} \\ 3) & e_{1}, & e_{2}-e_{3}, & e_{2}+e_{3} \\ 4) & e_{1}-e_{2}, & e_{1}+e_{2}, & e_{3}-e_{4}\end{array}$

Лемма 5. Тетраэдр $H_{4}$ не разбивает кубов Ламберта.

ДокАЗАтЕльство аналогично доказательству леммы 4. Рассмотрим $S^{3}$ как единичную сферу в $\mathbb{E}^{4}$. Пусть $e_{1}, e_{2}, e_{3}, e_{4}$ - ортонормированный базис $\mathbb{E}^{4}$. Пусть $\Delta$ - множество единичных векторов, ортогональных зеркалам группы $G_{H_{4}}$ (в данном случае $\Delta$ не является системой корней). С точностью до движения $\mathbb{E}^{4}$ множество $\Delta$ состоит из следующих 120 векторов:

$$
\begin{array}{llll} 
\pm(1,0,0,0), \quad \pm(0,1,0,0), \quad \pm(0,0,1,0), & \pm(0,0,0,1), & \frac{1}{2}( \pm 1, \pm 1, \pm 1, \pm 1), \\
\frac{1}{4}( \pm 2, \pm x, \pm y, 0), & \frac{1}{4}( \pm x, \pm y, \pm 2,0), & \frac{1}{4}( \pm y, \pm 2, \pm x, 0), & \frac{1}{4}( \pm x, \pm 2,0, \pm y), \\
\frac{1}{4}( \pm y, \pm x, 0, \pm 2), & \frac{1}{4}( \pm 2, \pm y, 0, \pm x), & \frac{1}{4}( \pm 2,0, \pm x, \pm y), & \frac{1}{4}( \pm x, 0, \pm y, \pm 2), \\
\frac{1}{4}( \pm y, 0, \pm 2, \pm x), & \frac{1}{4}(0, \pm x, \pm 2, \pm y), & \frac{1}{4}(0, \pm y, \pm x, \pm 2), & \frac{1}{4}(0, \pm 2, \pm y, \pm x),
\end{array}
$$

где $x=1+\sqrt{5}, y=1-\sqrt{5}$.

Для доказательства леммы достаточно проверить, что не существует шестерки векторов $f_{1}, \ldots, f_{6} \in \Delta$, удовлетворяющей условиям (1)-(4) из доказательства леммы 4.

Любые три взаимно ортогональных вектора из $\Delta$ совпадают с $\left\{e_{1}, e_{2}, e_{3}\right\}$ с точностью до движения из $G_{H_{4}}$. Несложно проверить с помощью условия (4), что куб Ламберта можно задать только с помощью следующих шестерок векторов $\left\{f_{1}, \ldots, f_{6}\right\}$ :

$$
\begin{aligned}
& \left\{\varepsilon_{1} e_{1}, \varepsilon_{2} e_{2}, \varepsilon_{3} e_{3},(x, 2,0, y), \varepsilon_{5}(0,-y, x, 2), \varepsilon_{6}(y, 0,2,-x)\right\}, \\
& \left\{\varepsilon_{1} e_{1}, \varepsilon_{2} e_{2}, \varepsilon_{3} e_{3},(y, x, 0,2), \varepsilon_{5}(0,2, y,-x), \varepsilon_{6}(2,0,-x,-y)\right\}, \\
& \left\{\varepsilon_{1} e_{1}, \varepsilon_{2} e_{2}, \varepsilon_{3} e_{3},(2, y, 0, x), \varepsilon_{5}(0, x, 2,-y), \varepsilon_{6}(x, 0,-y,-2)\right\}, \\
& \left\{\varepsilon_{1} e_{1}, \varepsilon_{2} e_{2}, \varepsilon_{3} e_{3},(y, x, 0,2), \varepsilon_{5}(0, y, x, 2), \quad \varepsilon_{6}(x, 0, y, 2)\right\},
\end{aligned}
$$

где $\varepsilon_{i}= \pm 1$ и $\left(t_{1}, t_{2}, t_{3}, t_{4}\right)=t_{1} e_{1}+t_{2} e_{2}+t_{3} e_{3}+t_{4} e_{4}$.

Никакая из этих шестерок не удовлетворяет условиям (2) и (3).

Леммы 3-5 доказьвают теорему 1.

2. Евклидовы кубы Ламберта. Пусть $P$-куб Ламберта в евклидовом пространстве $\mathbb{E}^{3}$ и $f$-грань куба $P$. Из определения куба Ламберта следует, что $f$ имеет не менее трех прямых углов. Поскольку четырехугольник $f$ евклидов, все его углы прямые. Таким образом, все плоские углы куба $P$ прямые, а следовательно, прямыми являются и все двугранные углы. Итак, доказана 
ТЕОрема 2. Пусть $P$ - куб Ламберта в $\mathbb{E}^{3} u G_{P}$ - группа отражсений, порожденная кубом $P$. Тогда $P=Q(\pi / 2, \pi / 2, \pi / 2)$ u $G_{P}$ дискретна.

3. Гиперболические кубы Ламберта. Пусть $P$ - куб Ламберта в гиперболическом пространстве $\mathbb{H}^{3}$. В данном пункте доказана

Теорема 3. Пусть $P$ - куб Ламберта в $\mathbb{H}^{3}$ и $G_{P}$ - группа отражений, порожденная кубом $P$. Группа $G_{P}$ дискретна тогда и только тогда, когда куб Ламберта $P$ является многогранником Кокстера (m.е. $P=Q\left(\pi / k_{1}, \pi / k_{2}, \pi / k_{3}\right)$, где $\left.k_{i} \geqslant 3, \quad i=1,2,3\right)$.

ДокАЗАТЕЛЬСтво. Предположим, что куб $P$ является многогранником Кокстера. Тогда группа $G_{P}$ дискретна и $P$ является ее фундаментальной областью. Гиперболический куб Ламберта $P=Q\left(\pi / k_{1}, \pi / k_{2}, \pi / k_{3}\right)$ существует тогда и только тогда, когда $k_{1}, k_{2}, k_{3} \geqslant 3$ (см. [7]). Таким образом, в случае кокстеровских кубов Ламберта теорема 3 очевидна.

В дальнейшем мы считаем, что куб $P$ не является кокстеровским, но группа $G_{P}$ дискретна. В этом случае $P$ допускает кокстеровское разбиение с некоторьп фундаментальным многогранником $F$.

Следующая лемма доказана в [4].

ЛЕмма 6. Пусть $P$ - ограниченный многогранник в $\mathbb{H}^{3}$, допускающий кокстеровское разбиение с фундаментальным многогранником F. Если $P$ - пирамида, то $F$ - тетраэдр. Если $P$ - треугольная призма, то $F$ - либо тетраэдр, либо треугольная призма.

ОБозначения. Пусть $A, B, C, D, A^{\prime}, B^{\prime}, C^{\prime}, D^{\prime}$ - вершины куба $P$. Поскольку $P$ куб Ламберта, все его ребра, кроме трех, образованы двумя взаимно ортогональньми гранями. Пусть $A A^{\prime}, B^{\prime} C^{\prime}$ и $C D$ - оставшиеся три ребра (см. рис. 2).

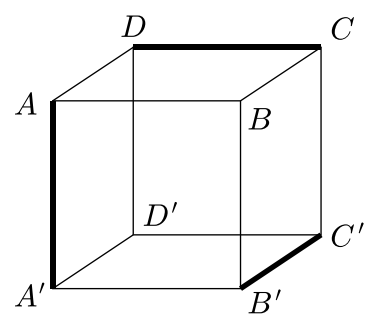

Рис. 2. Куб Ламберта

Лемма 7. Пусть $P$ - некокстеровский куб Ламберта в $\mathbb{H}^{3}$, допускающий кокстеровское разбиение. Тогда фундаментальный многогранник $F$ этого разбиения является либо тетраэдром, либо треугольной призмой.

ДокАЗАТЕльство. Поскольку куб $P$ не является кокстеровским, существует зеркало, разрезающее куб и проходящее через $A A^{\prime}, B^{\prime} C^{\prime}$ или $C D$. Пусть $\mu$ - зеркало, проходящее через $A A^{\prime}$. Зеркало $\mu$ резрезает $P$ на два многогранника. Если хотя бы один из них является треугольной призмой (см. рис. 3a), то лемма следует из леммы 6. 


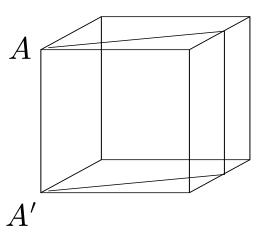

(a)

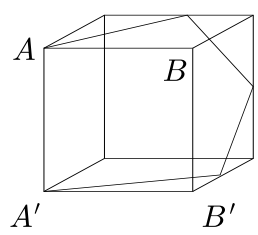

(b)

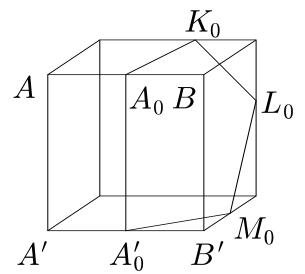

(c)

Рис. 3. Зеркала в кубе

Допустим, что ни одна из этих частей не является треугольной призмой (см. рис. $3 \mathrm{~b}$ ). Предположим, что существует зеркало $\mu^{\prime}$, разрезающее куб и проходящее через $B^{\prime} C^{\prime}$ или $C D$. Предположим, что $\mu^{\prime}$ не отрезает от $P$ треугольной призмы. Тогда $\mu$ и $\mu^{\prime}$ делят $P$ на четыре части, хотя бы одна из которых является либо тетраэдром, либо треугольной призмой (см. рис. 4). В этом случае лемма доказана.
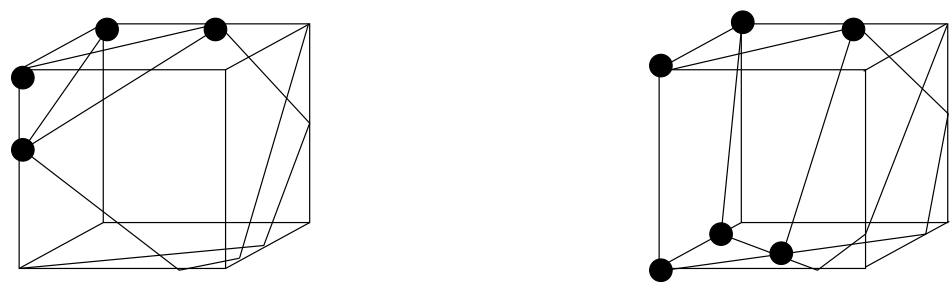

Рис. 4. Вершины тетраэдра и треугольной призмы (они обозначены жирными точками)

Предположим, что нет зеркала, проходящего через $B^{\prime} C^{\prime}$ или $C D$ и разрезающего куб. Тогда $\mu$ разрезает куб, как показано на рис. $3 \mathrm{~b}$. Рассмотрим многогранник $A^{\prime} A K L M B^{\prime} B C$. Рассмотрим множество $\Omega$ многогранников $P_{i}$, удовлетворяющих следующим свойствам:

1) $P_{i}$ лежит в $A^{\prime} A K L M B^{\prime} B C$;

2) все, кроме одной, грани многогранника $P_{i}$ являются гранями многогранника $P$; оставшаяся грань П является зеркалом;

$3)$ точки $B, B^{\prime}$ и $C$ - вершины $P_{i}$.

Множество $\Omega$ непусто $\left(A^{\prime} A K L M B^{\prime} B C \in \Omega\right), \Omega$ содержит конечное число многогранников. Следовательно, существует хотя бы один минимальныцй многогранник $P_{0} \in \Omega$, т.е. многогранник, не содержаший других многогранников $P_{i} \in \Omega$. Обозначим вершины многогранника $P_{0}$, как показано на рис. 3 c. Предположим, что существует зеркало $\mu^{\prime \prime}$, разрезающее $P_{0}$ и проходящее через ребро $A_{0}^{\prime} A_{0}, A_{0} K_{0}, K_{0} L_{0}, L_{0} M_{0}$ или $M_{0} A_{0}^{\prime}$. Тогда либо многогранник $P_{0}$ вопреки предположению не является минимальным, либо $\mu^{\prime \prime}$ отрезает от $P$ тетраэдр или треугольную призму. В последнем случае лемма следует из леммы 6 .

Предположим, что нет зеркала $\mu^{\prime \prime}$, разрезающего $P_{0}$ и проходящего через ребро $A_{0}^{\prime} A_{0}$, $A_{0} K_{0}, K_{0} L_{0}, L_{0} M_{0}$ или $M_{0} A_{0}^{\prime}$. Ранее мы предположили, что нет зеркала $\mu^{\prime}$, разрезающего $P$ и проходящего через $B^{\prime} C^{\prime}$ или $C D$. Двугранные углы при остальных ребрах многогранника $P_{0}$ прямые, поскольку $P$ - куб Ламберта. Таким образом, многогранник 
$P_{0}$ является кокстеровским, и следовательно, остроугольным. Грани $K_{0} L_{0} C$ и $A_{0}^{\prime} M_{0} B$ многогранника $P_{0}$ не пересекаются, но продолжения этих граней имеют общую прямую $C D$. Это противоречит остроугольности гиперболического многогранника $P_{0}$ (см. [8]).

Лемма 8. Пусть $P$ - некокстеровский куб Ламберта в $\mathbb{H}^{3}$, допускающий кокстеровское разбиение. Тогда фундаментальный многогранник $F$ этого разбиения является тетраәдром.

ДокАЗАТЕЛЬСТво. Предположим, что $F$ - треугольная призма. Пусть $\alpha$ и $\beta$ - основания призмы $F$. Рассмотрим цепочку фундаментальных призм $F_{i}$, лежащих внутри $P$, такую, что $F_{i}$ и $F_{i+1}$ имеют общее основание. Пусть $\bar{\alpha}_{i}$ и $\bar{\beta}_{i}$ - плоскости, содержащие основания призмы $F_{i}$. Никакие две из этих плоскостей не пересекаются, поскольку призма $F$ кокстеровская, и следовательно, остроугольная. Поэтому цепочка ограничена двумя взаимно противолежашими гранями куба $P$.

С помощью таких цепочек несложно показать, что две грани куба $P$ разбиты на треугольники (причем эти разбиения являются кокстеровскими). Оставшиеся четыре грани замощены четырехугольниками одним из способов, показанных на рис. 5 (иначе существует цепочка призм, ограниченная двумя смежньми гранями куба). Более подробные аргументы можно найти, например, в [4].
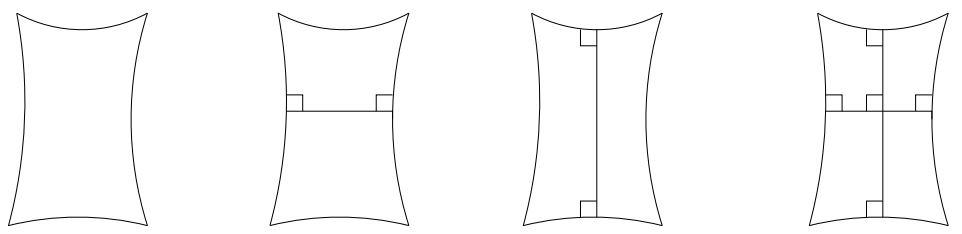

Рис. 5. Возможные разбиения граней куба

Пусть $A B C D$ и $A^{\prime} B^{\prime} C^{\prime} D^{\prime}$ - грани куба $P$, разрезанные на треугольники. Поскольку $P$ - куб Ламберта, оставшиеся грани не разрезаны (иначе получился бы гиперболический четырехугольник с четырьмя прямыми углами). Поэтому нет зеркал, разрезающих $P$ и проходящих через $B^{\prime} C^{\prime}$ или $C D$. Поскольку куб $P$ не кокстеровский, существует зеркало, разрезающее куб и проходящее через $A A^{\prime}$. Угол $\angle B A C$ грани $A B C D$ разрезан этим зеркалом. Остальные углы грани $A B C D$ прямые. Из [3] следует, что существует ровно два кокстеровских разбиения четырехугольника Ламберта с разрезанным острым углом. Эти разбиения показаны на рис. 6. Таким образом, разбиение грани $A B C D$ симметрично относительно $A C$, а грани $A^{\prime} B^{\prime} C^{\prime} D^{\prime}$ - относительно $A^{\prime} C^{\prime}$. Ясно, что в этом случае куб $P$ симметричен относительно плоскости $A A^{\prime} C^{\prime} C$.

Рассмотрим грань $A A^{\prime} B^{\prime} B$. Поскольку $P$ - куб Ламберта, $\angle B A^{\prime} A=\angle A A^{\prime} B=$ $\angle A B B^{\prime}=\pi / 2$. Угол $\angle B B^{\prime} A$ симметричен прямому углу $\angle D D^{\prime} A$ относительно $A A^{\prime} C^{\prime} C$. Следовательно, все углы четырехугольника $A A^{\prime} B^{\prime} B$ прямые, что невозможно.

$\mathrm{B} \mathbb{H}^{3}$ существует ровно девять ограниченных тетраэдров Кокстера. Список этих тетраэдров и обозначения приведены на рис. 7 .

Лемма 9. Пусть $P$ - некокстеровский куб Ламберта в $\mathbb{H}^{3}$, допускающий кокстеровское разбиение с фундаментальным тетраэдром $F$. Тогда $F=T_{6}$ или $F=$ $T_{7}$. 

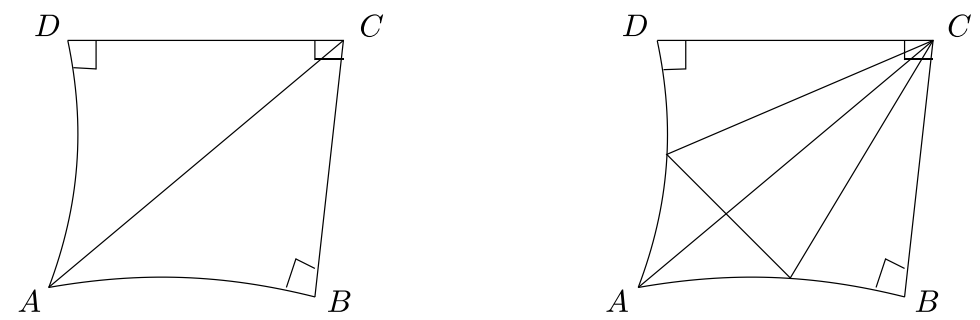

Рис. 6. Кокстеровские разбиения четырехугольников Ламберта

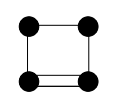

$T_{1}$

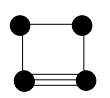

$T_{2}$

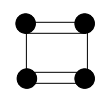

$T_{3}$

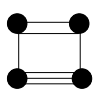

$T_{4}$

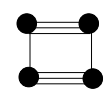

$T_{5}$

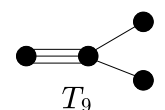

$T_{9}$

Рис. 7. Ограниченные гиперболические тетраэдры Кокстера. Схемы Кокстера и обозначения

ДокаЗАТЕльСтво. Поскольку куб $P$ не кокстеровский, существует зеркало $\mu$, разрезающее куб и проходящее через $A A^{\prime}, B^{\prime} C^{\prime}$ или $C D$. Будем считать, что $\mu$ содержит $A A^{\prime}$. Рассмотрим грань $A B C D$. Угол $\angle B A D$ равен $l \pi / k$, где $l, k \in \mathbb{Z}$ и $l \geqslant 2$. Остальные углы грани $A B C D$ прямые. Поскольку четырехугольник $A B C D$ гиперболический, сумма его углов меньше, чем $2 \pi$. Следовательно, $l / k<1 / 2$. Так как $\pi / k-$ двугранньй угол тетраэдра $F$, число $k$ является одним из чисел $2,3,4$ и 5 . Поэтому $l \pi / k=2 \pi / 5$, и один из двугранных углов тетраэдра $F$ равен $\pi / 5$.

Рассмотрим малую сферу $s$ с центром в точке $A$. Пересечение $s$ с кубом $P$ является треугольником $t$ с углами $2 \pi / 5, \pi / 2, \pi / 2$. Ограничение кокстеровского разбиения куба $P$ на сферический треугольник $t$ является кокстеровским разбиением треугольника $t$. Группа отражений, порожденная правильным прямоугольным треугольником $t$, не является подгруппой групы отражений, порожденной треугольником $(\pi / 2, \pi / 3, \pi / 5)$ (см. [6]). Поэтому $t$ разбит на две копии треугольника $(\pi / 5, \pi / 2, \pi / 2)$. Следовательно, пересечение $s$ с $F$ является треугольником $(\pi / 5, \pi / 2, \pi / 2)$, что возможно, только если $F=T_{6}$ или $F=T_{7}$.

Лемма 10. Некокстеровский куб Ламберта в $\mathbb{H}^{3}$ не допускает кокстеровского разбиения с фундаментальным тетраәдром $T_{6}$.

ДокАЗАТЕЛЬСтво. Предположим, что некокстеровский куб Ламберта $P$ допускает кокстеровское разбиение с фундаментальньм тетраэдром $T_{6}$. Тогда некоторая грань $f$ куба $P$ имеет углы $2 \pi / 5, \pi / 2, \pi / 2, \pi / 2$. Площадь этой грани равна $S_{f}=\pi(2-2 \pi / 5-$ $3 \pi / 2)=\pi / 10$.

Несложно проверить, что любой плоский угол любой грани тетраэдра $T_{6}$ либо меньше, чем $\pi / 4$, либо является прямым. Пусть $\alpha-$ грань $T_{6}$ с углом $\pi / 5$. Площадь грани $\alpha$ равна $S_{\alpha}=(\pi-\pi / 2-\pi / 5-\varphi)=3 \pi / 10-\varphi$, где $\varphi<\pi / 4$. Таким образом, $S_{\alpha}>\pi / 20$.

Поскольку грань $f$ куба $P$ имеет угол $2 \pi / 5, f$ содержит не менее двух образов гра- 
ни $\alpha$. Это невозможно, поскольку $S_{f}<2 S_{\alpha}$.

ЛЕмма 11. Некокстеровский куб Ламберта в $\mathbb{H}^{3}$ не допускает кокстеровского разбиения с фундаментальным тетраәдром $T_{7}$.

ДокАЗАТЕЛЬСТво. Предположим, что некокстеровский куб Ламберта $P$ допускает кокстеровскоеразбиение с фундаментальным тетраэдром $T_{7}$. Пусть $A A^{\prime}$ - ребро куба $P$, двугранный угол при котором равен $2 \pi / 5$. Тогда грань $A B C D$ имеет углы $2 \pi / 5, \pi / 2$, $\pi / 2, \pi / 2$. Площадь этой грани равна $S_{A B C D}=\pi(2-2 / 5-3 / 2)=\pi / 10$.

Рассмотрим ограничение кокстеровского разбиения на грань $A B C D$. Это замощение треугольниками, равньци граням тетраэдра $T_{7}$. Не менее двух треугольников в этом замошении имеют угол $\pi / 5$. Оставшиеся углы этих треугольников равны $\pi / 2$ и $\pi / 4$ (плоские углы тетраэдра $T_{7}$ легко вычислить в модели Клейна). Площадь треугольника $(\pi / 5, \pi / 2, \pi / 4)$ равна $\pi(1-1 / 2-1 / 4-1 / 5)=\pi / 20$. Поэтому $A B C D$ содержит ровно два треугольника, и существует зеркало, проходящее через точки $A^{\prime}, A, C$. По аналогичньм причинам это зеркало проходит через $C^{\prime}$. Отсюда следует, что куб Ламберта $P$ симметричен относительно плоскости $A^{\prime} A C C^{\prime}$, что невозможно (см. конец доказательства леммы 8).

Леммы 10 и 11 завершают доказательство теоремы 3.

Работа написана в университете г. Фрибур, Швейцария. Автор благодарит проф. Р. Келлерхалс и университет за гостеприимство. Автор благодарит проф. А. Д. Медных за постановку задачи.

\section{СПИСОК ЦИТИРОВАННОЙ ЛИТЕРАТУРЫ}

[1] Schwarz H. A. Über diejenige Fälle in welchen die Gaussische Reihe eine algebraische Function ihres elementes destellt // Crelle's J. 1873. V. 75. P. 292-335.

[2] Klimenko E., Sakuma M. Two-generator discrete subgroups of $\operatorname{Isom}\left(H^{2}\right)$ containing orientation-reversing elements // Geom. Dedicata. 1998. V. 72. P. 247-282.

[3] Felikson A. Coxeter decompositions of hyperbolic polygons // Europ. J. Combinatorics. 1998. V. 19. P. 801-817.

[4] Felikson A. Coxeter decompositions of hyperbolic pyramids and triangular prisms. math.MG/ 0212195 .

[5] Феликсон А. Кокстеровские разбиения гиперболических симплексов // Матем. сб. 2002. T. 193. №12. C. 134-156.

[6] Coxeter H. S. M. Finite groups generated by reflections, and their subgroups generated by reflections // Proc. Cambridge Philos. Soc. 1934. V. 30. P. 466-482.

[7] Андреев Е. М. О выпуклых многогранниках в пространствах Лобачевского // Матем. сб. 1970. T. 81. № 3. C. 445-478.

[8] Андреев Е. М. О пересечении плоскостей граней многогранников с острыми углами // Матем. заметки. 1970. Т. 8. №4. С. 521-527.

Независимый Московский университет

Поступило

E-mail: felikson@mccme.ru

21.06 .2002

Исправленный вариант

03.12 .2002 